\title{
Desempeño motor y hábitos de actividad física en estudiantes universitarios en Costa Rica
}

\author{
Motor performance and physical activity habits of college students in Costa Rica
}

\author{
Judith Jiménez-Díaz y María Morera-Castro ${ }^{2}$ \\ Escuela de Educación Física y Deportes, Universidad de Costa Rica ${ }^{l}$ \\ Escuela de Ciencias del Movimiento Humano y Calidad de Vida, Universidad Nacional, Costa Rica ${ }^{2}$ \\ judith.jimenez_d@ucr.ac.cr ${ }^{l}$
}

\begin{abstract}
Resumen
El objetivo del presente estudio fue analizar el desempeño motor de los patrones básicos de movimiento y los hábitos de actividad física del estudiantado de la Universidad de Costa Rica. Se evaluó a 92 hombres y 48 mujeres ( $\mathrm{M}=19.78$ años; $\mathrm{DE}=4.72$ años) matriculados en diferentes cursos de Actividad Deportiva de la Sede Rodrigo Facio. Se utilizó el Instrumento para la Evaluación de los Patrones Básicos de Movimiento para evaluar el desempeño motor en ocho patrones básicos de movimiento (correr, saltar, galopar, brincar, apañar, lanzar, rebotar y patear). El nivel de actividad física se obtuvo por medio de un cuestionario de autoreporte diseñado para dicho fin. Los resultados sugieren que el $28 \%$ del estudiantado presenta un estilo de vida físicamente activo. Los estudiantes presentan un desempeño maduro en los patrones de patear, correr y galopar. Por el contrario, se encontró que presentan un desempeño motor no maduro en los patrones de saltar, brincar, rebotar, lanzar y apañar. El nivel de actividad física está relacionado con el desempeño motor total de los participantes $(\mathrm{Rho}=.233 ; \mathrm{p}=.006$ ). Se concluye que los estudiantes universitarios presentan un desempeño eficiente en tres de ocho patrones evaluados; además, se encontró que el desempeño está relacionado con el nivel de actividad física reportado. Se recomienda a los profesionales en Ciencias del Movimiento Humano desarrollar actividades para fortalecer los patrones básicos de movimiento en los estudiantes universitarios.
\end{abstract}

Palabras clave: actividad física, patrones fundamentales, adultos-jóvenes, sedentarismo.

\begin{abstract}
The purpose of this study was to analyze the motor performance of fundamental motor skills and physical activity habits of students at the University of Costa Rica. A total of 92 males and 48 females ( $\mathrm{M}$ age $=19.78$ yr., $\mathrm{SD}=4.72$ yr.) enrolled in different Sports Activity courses taught at the Rodrigo Facio campus was assessed. The Instrument for the Evaluation of Fundamental Movement Patterns was used to assess motor performance in eight fundamental movement patterns (running, jumping, galloping, catching, throwing, bouncing, and kicking). The physical activity level was obtained from a self-reported questionnaire developed for such purpose. Results show that $28 \%$ of the participants were physically active. Participants presented a proficient performance in kicking, running, and galloping, but a non-proficient performance in jumping, hopping, bouncing, throwing and catching. Physical activity behavior was related to the overall performance of the motor skills assessed $(\mathrm{Rho}=.233 ; \mathrm{p}=.006)$. In conclusion, college students presented a proficient performance on three of the eight skills assessed. In addition, a relationship was found between physical activity levels and performance. Physical Education teachers are recommended to develop activities to enhance motor performance of fundamental motor skills in college students.
\end{abstract}

Keywords: physical activity, fundamental motor skills, young adults, sedentary behavior. 


\section{Introducción}

La Organización Mundial de la Salud (OMS) indicó que en el mundo mueren 1.9 millones de personas al año a causa de la inactividad física (OMS, 2010). El sedentarismo está definido como el nivel de actividad física insuficiente, caracterizado por realizar menos de 150 minutos de actividad física por semana y está asociado a muerte por cáncer (pulmonar, colon, estómago, esófago, mama y endometrio), enfermedades cerebrovasculares, enfermedad isquémica del corazón, enfermedad hipertensiva, enfermedad pulmonar, diabetes tipo 2 y otras causas cardiovasculares (ACSM, 2011; Ministerio de Salud, 2010). En Costa Rica, la información completa más reciente indica que para el año 2005 la prevalencia del sedentarismo fue de un $32.70 \%$ en personas con edades entre los 15 y 29 años, de un $30.40 \%$ en personas con edades entre 30 y 44 años y de un $35.70 \%$ en personas con edades entre los 45 y 59 años (Ministerio de Salud, 2010).

En un estudio realizado en tres universidades públicas del país en el año 2011 (UNED, TEC y UNA) se concluyó que el $51.7 \%$ de los estudiantes de primer ingreso $(n=7868)$ son sedentarios, mientras que el $45.3 \%$ realizan actividad física tres veces por semana en una actividad moderada (Trejos y Solís, 2011). Para ese mismo año -2011- en la Universidad de Costa Rica se reportó que el $24.06 \%$ de los estudiantes de primer ingreso $(n=4251)$ nunca realizan actividad física, el $37.33 \%$ realiza actividad física una o dos veces por semana, el $18.30 \%$ tres días por semana y $20.3 \%$ cuatro o más días por semana. Al tomar en cuenta que realizar actividad física una o dos veces por semana no se considera ser físicamente activo, se puede interpretar que el $61.39 \%$ de los estudiantes de primer ingreso para el año 2011 en la UCR presentó un estilo de vida sedentario; por el contrario, el $38.60 \%$ era físicamente activo (Oficina de Bienestar y Salud, 2011).

Aunque no se conocen con certeza las razones que incitan a las personas a realizar actividad física, Stodden et al. (2008) indican que un buen dominio de los patrones básicos de movimiento es un factor importante que aumenta las probabilidades de ser físicamente activo. Esto porque una persona tiene más opciones de participar en diferentes actividades físicas cuando logra ejecutar distintas destrezas motrices. Los patrones básicos de movimiento son los gestos motrices simples involucrados en el desarrollo motor grueso, fundamentales para poder ejecutar movimientos más complejos, utilizados en una gran variedad de actividades físicas y en deportes específicos (Gabbard, 2012; Gallahue y Ozmun, 2005; Hardy, Reinten-Reynolds, Espinel, Zask, y Okely, 2012; Morgan et al., 2013; Stodden et al., 2008).

Los patrones básicos de movimiento (PBM) comúnmente se clasifican en patrones locomotores o manipulativos. Los patrones locomotores sirven para desplazar el cuerpo de un lugar al otro; los ejemplos más comunes son caminar y correr, pero existen otros movimientos como saltar, brincar, galopar, entre muchos más. Los patrones manipulativos sirven para proyectar o recibir objetos; por ejemplo, lanzar, apañar, patear, rebotar una bola, entre otras (Gabbard, 2012; Logan, Robinson, Wilson y Lucas, 2012). 
La etapa madura de un gesto se caracteriza por movimientos mecánicamente eficientes y un desempeño coordinado y controlado (Gallahue y Ozmun, 2005). Düger et al. (1999) mencionan que, en promedio, a los seis años de edad los niños(as) alcanzan el grado de maestría (etapa madura), pero una gran mayoría no supera la etapa elemental del gesto. Clark (2007) considera que es necesario adquirir el patrón maduro para realizar diferentes actividades físicas a lo largo de la vida. Lamentablemente, adquirir el patrón maduro -desempeño eficiente, fluido y coordinado del movimiento- de diferentes destrezas no se logra de forma natural, requiere de la enseñanza, el aprendizaje y la práctica del movimiento (Gabbard, 2012; Hardy et al., 2012; Morgan et al., 2013). Adicionalmente, en estudios recientes se ha identificado que muchas personas no alcanzan un patrón eficiente (maduro) de las destrezas simples (Hardy et al., 2012; Stodden, True, Langendorfer y Gao, 2013). Al considerar la importancia de ser físicamente activo y presentar un desempeño motor eficiente, en este estudio se planteó como objetivo describir el desempeño motor de los patrones básicos de movimiento y los hábitos de actividad física de los estudiantes del curso Actividad Deportiva de la Universidad de Costa Rica.

\section{Metodología}

\section{Participantes}

Un total de 48 mujeres y 92 hombres ( $n=140$; edad $M=19.78 \pm 4.72$, peso $M=64.20 \pm$ 10.99 , talla $M=167.67 \pm 7.97$ e índice de masa corporal $M=22.81 \pm 3.41$ ) matriculados en diferentes cursos de Actividad Deportiva de la Universidad de Costa Rica participaron voluntariamente del estudio y firmaron el consentimiento informado respectivo. El estudiantado está empadronado en diversas carreras, entre las más frecuentes se encuentran: Ingeniería (con diferentes énfasis), Administración de Empresas, Enseñanza (con diferentes énfasis) y Derecho.

\section{Instrumentos}

El desempeño motor de los estudiantes se evaluó utilizando el Instrumento de Evaluación de los Patrones Básicos de Movimiento (IPBM) (Jiménez, Salazar y Morera, 2013). Este instrumento es una prueba orientada al proceso y evalúa el desempeño de cada patrón en su etapa madura. Cada patrón se describe con seis características observables, el participante recibe un punto si muestra la característica y cero puntos si no la presenta. El puntaje posible a obtener va de 0 a 12 puntos por patrón. La subescala de locomoción y manipulación es el promedio de los patrones evaluados en cada componente. El total del desempeño se obtiene con el promedio de todos los patrones evaluados. Un promedio de nueve puntos o más indica un desempeño eficiente (patrón maduro). El IPBM presenta una validez de contenido dada por el juicio de expertos, además de coeficientes de confiabilidad y objetividad intra-clase de $R=0.918$ y $R=$ 
0.861, respectivamente. El IPBM evalúa 10 patrones básicos (correr, galopar, brincar, lanzar, saltar, deslizar, batear, apañar, patear y rebotar); para este estudio se utilizaron ocho escalas (correr, galopar, brincar, lanzar, saltar, apañar, patear y rebotar).

Con el propósito de describir la muestra, se administró un cuestionario para conocer las características demográficas de los estudiantes, en este se preguntó sobre la carrera en la que se encuentra empadronada, la cantidad de días que realiza actividad física a la semana, el tipo de actividad física que práctica y la fecha de nacimiento. Adicionalmente, se utilizó un tallímetro portátil para medir la talla y una báscula (BF-682W TANITA) para obtener el peso (los valores se redondearon al decimal 5 más cercano); el índice de masa corporal (IMC) se obtuvo con la fórmula: $\mathrm{IMC}=\mathrm{kg} / \mathrm{m}^{2}$.

\section{Procedimiento}

En diferentes cursos de Actividad Deportiva de la Universidad de Costa Rica se le solicitó al estudiantado su participación voluntaria en el estudio. Luego de una explicación del estudio y de los instrumentos a utilizar, cada persona contestó el cuestionario suministrado. Posteriormente, los participantes fueron pesados y medidos; una vez obtenidos el peso y la talla, se calculó el IMC. A continuación se realizó la medición de los patrones básicos de movimiento, siguiendo las indicaciones del IPBM. Se filmó a cada persona ejecutando cada patrón en dos intentos y la evaluación del desempeño motor se realizó a partir del video, siguiendo los procedimientos establecidos.

\section{Análisis estadístico}

Se obtuvo el promedio, desviación estándar (variables categóricas) y frecuencias (variables categóricas) utilizando un programa de hoja de cálculo (Microsoft Excel 2013). Se realizó una correlación de Spearman entre el desempeño motor total y los hábitos de actividad física del estudiantado, utilizando el programa estadístico IBM-SPSS versión $21 \circledast$. Los valores con un alfa menor o igual a 0.05 se consideran estadísticamente significativos.

\section{Resultados}

En la Tabla 1 se muestran los valores descriptivos del desempeño motor por patrón de las personas participantes en el estudio. Los resultados señalan que en promedio las personas participantes presentaron un puntaje mayor a nueve puntos en los patrones de correr, galopar y patear. Sin embargo, se obtuvo un puntaje menor a nueve en los patrones de lanzar, apañar, saltar, brincar, rebotar. En las subescalas, se obtuvo un promedio menor a nueve en los patrones manipulativos (lanzar, apañar, rebotar y patear) y un promedio menor a nuevo en los patrones locomotores (correr, galopar, saltar y brincar). En el total del desempeño (promedio de los ocho patrones) se encontró un puntaje menor a nueve (ver Figura 1). 
URL: http://www.revistas.una.ac.cr/mhsalud

Tabla 1

Estadística descriptiva del desempeño motor por patrón de las personas participantes

\begin{tabular}{lccc}
\hline \multicolumn{1}{c}{ Desempeño Motor } & $\mathrm{M}$ & $\mathrm{DE}$ & Rango \\
\hline Correr & 9.39 & 1.61 & $5-12$ \\
Galopar & 9.24 & 1.73 & $4-12$ \\
Saltar a distancia & 8.57 & 1.75 & $4-12$ \\
Brincar & 7.80 & 2.79 & $1-12$ \\
Total de locomoción & 8.75 & 1.31 & $5.5-11.5$ \\
Rebotar & 8.55 & 2.34 & $0-12$ \\
Lanzar & 5.17 & 2.77 & $0-12$ \\
Apañar & 8.85 & 2.25 & $0-12$ \\
Patear & 9.09 & 3.01 & $0-12$ \\
\hline Total de manipulación & 7.92 & 1.92 & $0-11.75$ \\
\hline Total de desempeño & 8.24 & 1.74 & $0-11.38$ \\
\hline
\end{tabular}

Nota: datos se presentan en media (M), desviación estándar (DE) y rango.

El $57 \%$ de las personas participantes indicó realizar actividad física una o dos veces por semana, el $22 \%$ la realiza de tres a cuatro veces, mientras que el $6 \%$ la realiza más de cinco veces a la semana. Un $15 \%$ del grupo encuestado no realiza actividad física de manera regular (ver Figura 2). Entre las actividades que los estudiantes aseguran realizar con mayor frecuencia se encuentran salir a correr, jugar fútbol con amigos, jugar baloncesto con amigos, ir al gimnasio (hacer pesas), andar en bicicleta y clases de aeróbicos.

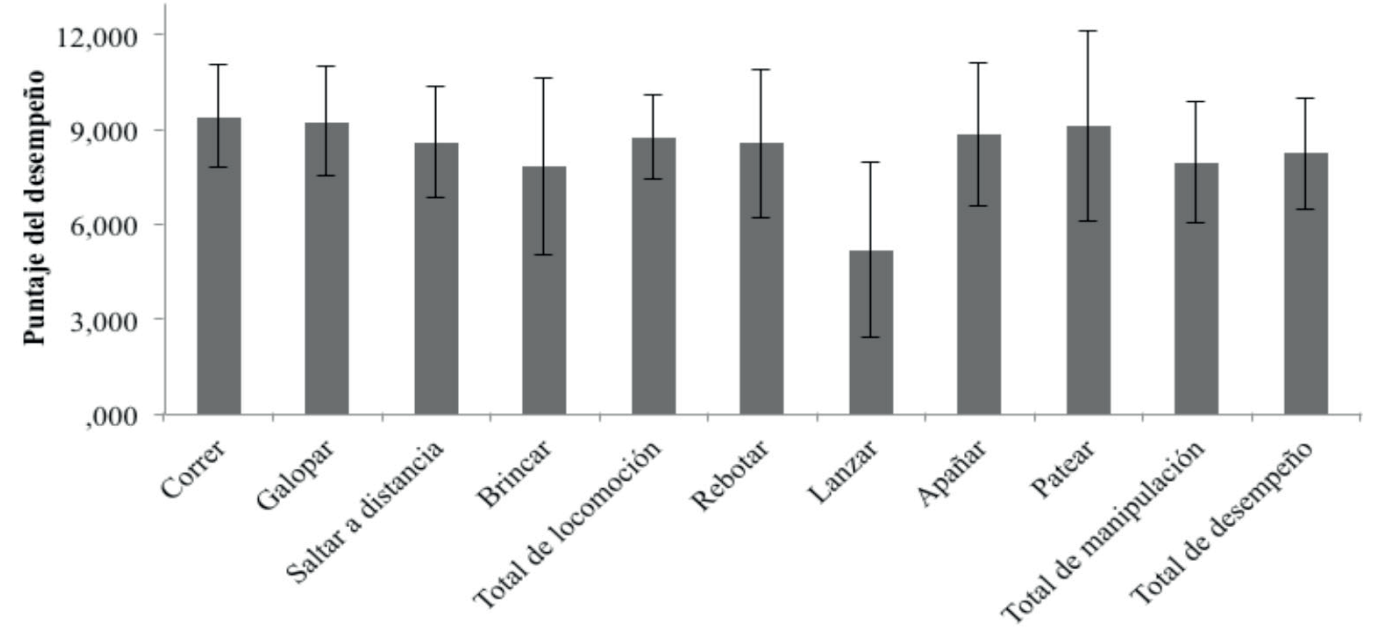

Patrones básicos de movimiento

Figura 1. Desempeño motor de las personas participantes 
URL: http://www.revistas.una.ac.cr/mhsalud

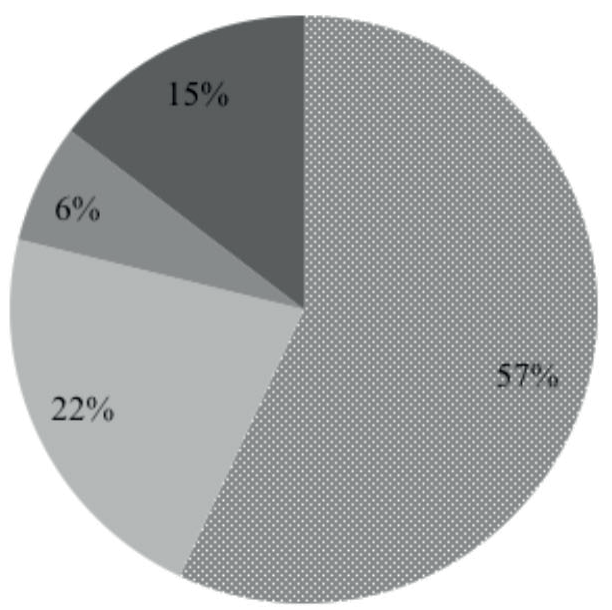

Actividad física 1 o 2 veces por semana

Actividad física 3 o 4 veces por semana

- Actividad física más de 5 veces por semana

- No realiza actividad física

Figura 2. Hábitos de actividad física de las personas participantes

Se encontró una relación positiva significativa entre el desempeño motor total y los hábitos de actividad física de las personas participantes $(R h o=.233 ; p=.006)$. Asimismo, se encontró una relación positiva con los patrones de saltar, correr y brincar y con el componente de locomoción. En la Tabla 2 se presentan las correlaciones para cada uno de los patrones evaluados y las subescalas.

Tabla 2

Relación de Spearman entre del desempeño motor y el nivel de actividad física por patrón

\begin{tabular}{lll}
\hline Desempeño motor & Rho & $p$ \\
\hline Correr & .217 & .011 \\
Galopar & .008 & .929 \\
Saltar a distancia & .316 & .001 \\
Brincar & .188 & .027 \\
Total de locomoción & .285 & .001 \\
Rebotar & .075 & .384 \\
Lanzar & .098 & .255 \\
Apañar & .003 & .970 \\
Patear & .152 & .076 \\
\hline Total de manipulación & .131 & .126 \\
\hline Total de desempeño & .233 & .006 \\
\hline
\end{tabular}




\section{Discusión}

El objetivo del presente estudio fue analizar el desempeño motor de los patrones básicos de movimiento y los hábitos de actividad física, en una muestra de estudiantes de cursos de Actividad deportiva de la Universidad de Costa Rica. Se obtuvo que el $72 \%$ de la población estudiantil estudiada realiza actividad física menos de dos veces por semana. El restante $28 \%$ es físicamente activo según los lineamientos del ACSM (2011). Se evidencia que la población estudiantil universitaria del presente estudio, presentan un factor de riesgo importante en su salud al no mantener hábitos saludables de actividad física (ACSM, 2011). Los datos de este grupo de universitarios concuerdan con los estudios realizados por las diferentes universidades públicas del país, en referencia a los niveles de actividad física de los estudiantes de primer ingreso para el año 2011 (Oficina de Bienestar y Salud, 2011; Trejos y Solís, 2011). Cabe resaltar que los datos indican altos niveles de sedentarismo, por lo que es importante promover cambios en los niveles de actividad física de la población estudiantil universitaria.

Con respecto al desempeño motor de los participantes, en general se encontró que presentan un desempeño deficiente (no maduro). Específicamente, en los patrones locomotores se presentó un desempeño eficiente en correr y galopar. Mientras que en los patrones manipulativos se mostró un desempeño eficiente en patear. Esto concuerda con las conclusiones de otros estudios que consideran normal que las personas no presenten un desempeño maduro de las destrezas simples (Hardy et al., 2012; Stodden et al., 2013).

El estudiantado físicamente activo, en su mayoría mencionó que preferían salir a correr o jugar fútbol con sus amigos, esto explica un mejor desempeño en los patrones de correr y patear, ya que estas son destrezas base para este tipo de actividades. Un nivel eficiente de desempeño motor se logra con la práctica y la instrucción (Morgan et al., 2013), por esto es razonable pensar que la participación en actividades como el fútbol promueven un mejor desempeño de los patrones de correr y patear, y a su vez incrementa los niveles de actividad física de las personas que las practican.

En el presente estudio se encontró una relación positiva significativa entre el nivel de actividad física realizada por semana y el desempeño motor total del estudiantado; es decir, a mejor desempeño en la ejecución de patrones básicos, mayor nivel de actividad física presentado. Esto resultados concuerdan con otros estudios similares que han encontrado una relación entre estas dos variables (Cohen, Morgan, Plotnikoff, Callister y Lubans, 2014; Logan et al., 2012; Spessato, Gabbard, Robinson y Valentini, 2012; Stodden y Goodway, 2007; Wrotniak, Epstein, Dorn, Jones y Kondilis, 2006). Específicamente, la relación se presenta en los patrones locomotores (correr, saltar y brincar), lo cual concuerda con otros estudios (Cohen et al., 2014; Spessato, Gabbard y Valentini, 2013).

Al tomar en cuenta que existe una relación significativa entre el desempeño motor y el nivel de actividad física, se puede asumir que un desempeño eficiente de los patrones básicos 
facilita la participación en actividades físicas, lo que promueve un nivel mayor de actividad física (Cohen et al., 2014; Wrotniak et al., 2006). Consecuentemente, es factible considerar que si en las lecciones de Educación Física o, en este caso, en los cursos de Actividad Deportiva de las universidades, se desarrollan actividades que promuevan el desempeño de los PBM, se podría aumentar el nivel de actividad física de la población estudiantil universitaria. Por tal motivo, es importante desarrollar estrategias para motivar a las personas a realizar actividad física y así reducir los niveles de sedentarismo en la población estudiada, para que perciba beneficios en su salud física y mental.

\section{Conclusiones y recomendaciones}

Los resultados del presente estudio indican que la población estudiantil universitaria estudiada presenta un desempeño eficiente en tres de ocho patrones evaluados. Además, un $28 \%$ de los estudiantes son físicamente activos, por lo que un alto porcentaje (72\%) presenta hábitos sedentarios. También se encontró que el desempeño motor total está positivamente relacionado con el nivel de actividad física de la población estudiantil; específicamente con los patrones locomotores (correr, saltar, brincar).

Con base en esta relación, se recomienda buscar e implementar estrategias que motiven a las personas adultas-jóvenes y a poblaciones de otras edades a mantener un estilo de vida físicamente activo y, por ende, un estilo de vida saludable. Una posible estrategia a emplear es realizar intervenciones que se enfoquen en mejorar el desempeño motor de los patrones básicos de movimiento, ya que las personas al sentirse más exitosas en su ejecución, podrían sentir mayor motivación para realizar actividad física; esto debido a que la presencia de un desempeño maduro facilita realizar otro tipo de movimientos motrices y promueve la participación en diferentes actividades físicas (Stodden et al., 2008).

\section{Referencias bibliográficas}

American College of Sports Medicine [ACSM]. (2011). Quantity and Quality of Exercise for Developing and Maintaining Cardiorespiratory, Musculoskeletal, and Neuromotor Fitness in Apparently Healthy Adults: Guidance for Prescribing. Exercise Medicine \& Science in Sports \& Exercise, 43(7):1334-1359. http://dx.doi.org/10.1249/MSS.0b013e318213fefb

Clark, J.E. (2007). On the problem of motor skill development. Journal of Physical Education, Recreation and Dance, 78(5), 39-44. http://dx.doi.org/10.1080/07303084.2007.10598023

Cohen, K. E., Morgan, P. J., Plotnikoff, R. C., Callister, R. \& Lubans, D. R. (2014). Fundamental movement skills and physical activity among children living in low-income communities: a cross-sectional study. International Journal of Behavioral Nutrition and Physical Activity, 11(1), 49-58. http://dx.doi.org/10.1186/1479-5868-11-49 
Düger, T., Bumin, G., Uyanik, M., Aki, E. \& Kayihan, H. (1999). The assesment of BruininksOseretsky test of motor proficiency in children. Pediatric Rehabilitation, 3 (3), 125-131.

Gabbard, C. P. (2012). Lifelong Motor Development (6 ${ }^{\text {th }}$ ed.). San Francisco: Benjamin-Cummings Publishing Company.

Gallahue, D. L. \& Ozmun, J. C. (2005). Understanding Motor Development: Infants, Children, Adolescents, Adults ( $6^{\text {th }}$ ed.): McGraw-Hill Higher Education.

Hardy, L. L., Reinten-Reynolds, T., Espinel, P., Zask, A. \& Okely, A. D. (2012). Prevalence and correlates of low fundamental movement skill competency in children. Pediatrics, 130(2), e390-e398. doi: 10.1542/peds.2012-0345

Jiménez, J., Salazar, W. y Morera, M. (2013). Diseño y validación de un instrumento para la evaluación de patrones básicos de movimiento. Motricidad. European Journal of Human Movement, 31(0), 87-97.

Logan, S. W., Robinson, L. E., Wilson, A. E. \& Lucas, W. A. (2012). Getting the fundamentals of movement: A meta-analysis of the effectiveness of motor skill interventions in children. Child: Care, Health and Development, 38(3), 305-315. doi: 10.1111/j.1365-2214.2011.01307.

Ministerio de Salud. (2010). Estimación de muertes atribuibles a principales factores de riesgo en Costa Rica, 2005. Recuperado de: https://www.ministeriodesalud.go.cr/

Morgan, P. J., Barnett, L. M., Cliff, D. P., Okely, A. D., Scott, H. A., Cohen, K. E. \& Lubans, D. R. (2013). Fundamental Movement Skill Interventions in Youth: A Systematic Review and Meta-analysis. Pediatrics, 132(5), e1361-e1383. http://dx.doi.org/10.1542/peds.2013-1167

Oficina de Bienestar y Salud, Universidad de Costa Rica [OBS]. (2011). Análisis de situación integral de salud de los estudiantes de primer ingreso 2011 de la Oficina de Bienestar y Salud. Comunicación personal con el Dr. Saúl Aguilar Morales (6 de junio, 2012).

Organización Mundial de la Salud [OMS]. (2010). La Organización Mundial de la Salud y el Comité Olímpico Internacional firman un acuerdo para fomentar los modos de vida sanos. Recuperado de: http://www.who.int/mediacentre/news/releases/2010/ioc_20100721/es/

Spessato, B., Gabbard, C., Robinson, L. \& Valentini, N. (2012). Body mass index, perceived and actual physical competence: The relationship among young children. Child: Care, Health and Development, 39(6), 845-850. http://dx.doi.org 10.1111/cch.12014

Spessato, B., Gabbard, C. \& Valentini, N. C. (2013). The role of motor competence and body mass index in children's activity levels in physical education classes. Journal of Teaching in Physical Education, 32(2), 118-130. 
Stodden, D. F. \& Goodway, J. D. (2007). The dynamic association between motor skill development and physical activity. Journal of Physical Education, Recreation \& Dance, 78(8), 33-49. http://dx.doi.org/10.1080/07303084.2007.10598077

Stodden, D. F., Goodway, J. D., Langendorfer, S. J., Roberton, M. A., Rudisill, M. E., Garcia, C. \& Garcia, L. E. (2008). A developmental perspective on the role of motor skill competence in physical activity: An emergent relationship. Quest, 60(2), 290-306. doi: http://dx.doi.org $\underline{10.1080 / 00336297.2008 .10483582}$

Stodden, D. F., True, L. K., Langendorfer, S. J. \& Gao, Z. (2013). Associations among selected motor skills and health-related fitness: indirect evidence for Seefeldt's proficiency barrier in young adults? Research Quarterly for Exercise and Sport, 84(3), 397-403. http://dx.doi. org/10.1080/02701367.2013.814910

Trejos, C. y Solís, M. (2011) Estilos de vida en Costa Rica: estudiantes universitarios de primer ingreso.

Wrotniak, B. H., Epstein, L. H., Dorn, J. M., Jones, K. E. \& Kondilis, V. A. (2006). The relationship between motor proficiency and physical activity in children. Pediatrics, 118(6), e1758-e1765. http://dx.doi.org/10.1542/peds.2006-0742

Recepción: 16 de junio del 2015

Corrección: 15 de octubre del 2015

Aceptación: 21 de noviembre del 2016

Publicación: 29 de enero del 2016

1 Judith Jiménez-Díaz es Máster en Ciencias del Movimiento Humano por la Universidad de Costa Rica y se desempeña como académica en la Escuela de Educación Física y Deportes, Universidad de Costa Rica.

Maria Morera-Castro es Ph.D en Kinesiología con énfasis en comportamiento motor de la Universidad de Auburn, USA. Académica de la Escuela de Ciencias del Movimiento Humano y Calidad de Vida, Universidad Nacional. 